\title{
The Atomic-level Characterization of Platinum Nanoparticles in $\gamma$-Alumina using TEM and DFT
}

Arielle Clauser ${ }^{1 *}$, Kofi Oware Sarfo ${ }^{1}$, Colin Ophus ${ }^{2}$, Raquel Giulian ${ }^{3}$, Líney Árnadóttir ${ }^{1}$, and Melissa K. Santala ${ }^{1}$

1. Oregon State University, Material Science and Engineering, Corvallis, USA.

2. National Center for Electron Microscopy, Lawrence Berkeley National Laboratory, Berkeley, USA.

3. Universidade Federal do Rio Grande do Sul, Porto Alegre, Brazil

*clausera@oregonstate.edu

Understanding metal/metal oxide interfaces is important for variety of modern devices and processes that depend on these heterointerface systems. Gamma alumina is metal oxide that is used as a support for metallic nanoparticles and is a key material in catalysis due to its high specific surface area and Lewis sites. ${ }^{1}$ The microstructure of gamma alumina has been the subject of numerous experimental and theoretical studies due to the catalytic significance of this metastable alumina phase. ${ }^{2,3}$ The goal of this work is to gain atomic-level structural information of the $\mathrm{Pt} / \gamma$-interfaces through the comparison of experimentally determined results and density functional theory (DFT) calculations..

Model $\gamma$-alumina microstructures are formed using high energy ion implantation of Pt into single crystal $\alpha$-alumina wafers followed by thermal annealing. ${ }^{4}$ Crystallization of amorphized alumina proceeds through the metastable phase series $\gamma \rightarrow \delta \rightarrow \theta \rightarrow \alpha-\mathrm{Al}_{2} \mathrm{O}_{3}$ with increasing temperature. ${ }^{5}$ Choice of the annealing temperature allows for control of the re-crystallized matrix phase. Platinum-implanted $\mathrm{Al}_{2} \mathrm{O}_{3}$ annealed at $800^{\circ} \mathrm{C}$ provides $\mathrm{Pt} / \gamma$-alumina interfaces in the form of faceted metallic platinum nanoparticles embedded in a $\gamma$-alumina matrix. Characterization using X-ray diffraction confirmed the phase of alumina wafers post annealing. Characterization with transmission electron microscopy (TEM) provides more crystallographic data and high resolution structural data. Paired with density functional theory (DFT) simulations this provides a path toward a deeper understanding of the atomic level Pt/ $\gamma$ alumina interfacial structure.

The recrystallization of implanted alumina occurs epitaxially to the underlying undamaged $\alpha$-alumina and results in the alignment close-packed $\mathrm{O}$ planes of $(0006)_{\alpha} \|(111)_{\gamma}$ as determined using selected area electron diffraction and aberration-corrected high-resolution TEM. Fig. 1 shows a bright-field/dark-field pair of faceted Pt nanoparticles with a $(111)_{\mathrm{Pt}} \|(111)_{\gamma}$ orientation. Nanoparticles at high resolution surveyed had (111) $\mathrm{Pt}$ and (001) $\mathrm{Pt}$ oriented interfaces as shown in Fig. 2. The observed faceting was the basis for DFT interfacial structure calculations of possible chemical terminations shown in Fig 3, which will be further compared to experimental data. 


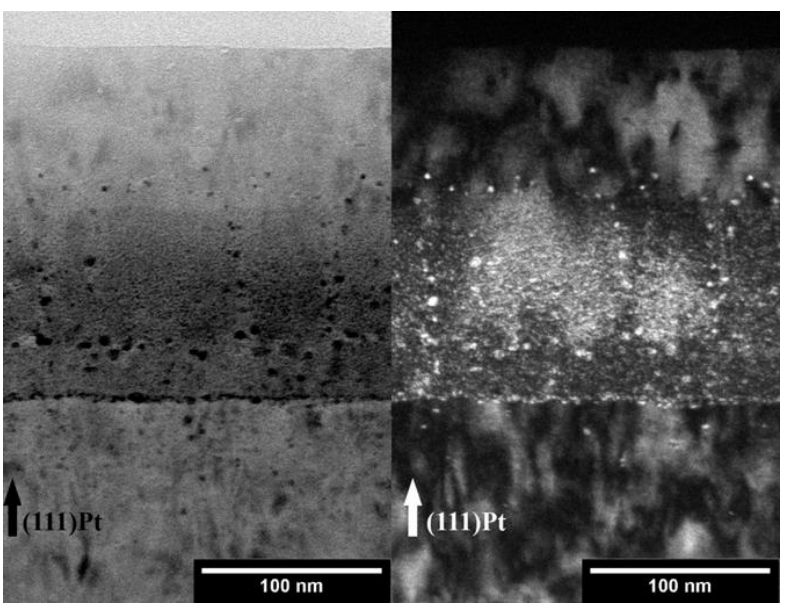

Figure 1. Two-beam bright-field/dark-field TEM image showing embedded Pt nanoparticles with (111)Pt $\|(111) \gamma$ orientation.

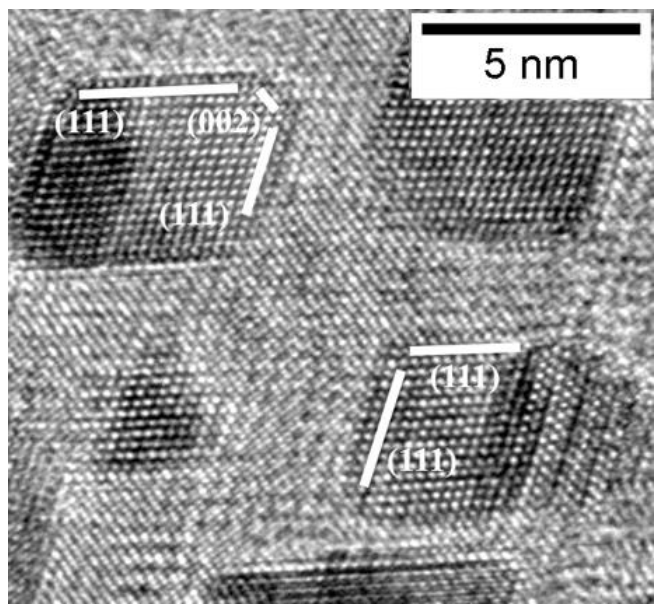

Figure 2. HRTEM image of embedded platinum nanoparticles present predominantly (111) facets confirming DFT calculations.

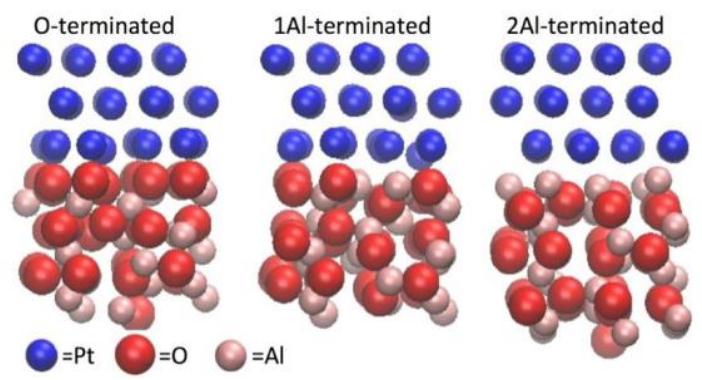

Figure 3. DFT simulation of possible terminations of $\mathrm{Pt} / \gamma-\mathrm{Al}_{2} \mathrm{O}_{3}$ interfaces

References:

[1] Trueba, M., \& Trasatti, S. P. (2005). $\gamma$-alumina as a support for catalysts: A review of fundamental aspects. European Journal of Inorganic Chemistry, (17), 3393-3403.

[2] Zhou, R.-S., \& Snyder, R. L. (1991). Structures and transformation mechanisms of the eta, gamma, and theta transitions aluminas. Acta Crystallographica, 47(5), 617-630

[3] Kovarik, L., Genc, A., Wang, C., Qiu, A., \& Peden, C. H. F. (2013). Tomography and HighResolution Electron Microscopy Study of Surfaces and Porosity in a Plate-like $\gamma$ - Al 2 O 3. The Journal of Physical Chemistry C, 117, 179-186.

[4] White, C. W., McHargue, C. J., Sklad, P. S., Boatner, L. A., \& Farlow, G. C. (1989). Ion implantation and annealing of crystalline oxides. Materials Science Reports, 4(2), 41-146.

[5] Wefers and C. Misra, "Oxides and Hydroxides of Aluminum: Alcoa Technical Paper No. 19, Revised ": Alcoa Laboratories, (1987)

[6] The authors acknowledge funding from National Science Foundation Grant No. 1610507. Electron microscopy was performed at the Oregon State University Electron Microscopy Facility (supported by NSF MRI 1040588, the Murdock Charitable Trust, and the Oregon Nanoscience and Micro-Technologies Institute) and at the Center for Advances Materials Characterization in Oregon facility at the University of Oregon. Work at the Molecular Foundry was supported by the Office of Science, Office of Basic Energy Sciences, of the U.S. Department of Energy under Contract No. DE-AC02-05CH11231. 